\title{
Design and development of coil casing MRF brake system
}

\author{
R. Siti Lydia ${ }^{1, *}, R$. Mokhtar ${ }^{1}, A . B$. Muhamad Husaini ${ }^{1}$ and B.J. Norhaniza ${ }^{1}$ \\ ${ }^{1}$ System Engineering and Energy Laboratory, Section of Manufacturing, Universiti Kuala Lumpur \\ Malaysian Spanish Institute, Kulim Hi-Tech Park, 09000 Kulim, Kedah.
}

\begin{abstract}
Nowadays magneto-rheological brakes has been introduce to overcome the drawback of conventional braking such as bulky, leakage and late build up pressure. Magnetic field is a main parameter need to be maximized in order to increase the efficiency of the brake. In MRF brake system the magnetic field directly affected by coil casing shapes. This paper focused on the study of the effect of coil casing shapes to the magnetic field distribution in MR brake. Finite Element Method will be used to analyze the magnetic field distribution produced by the coil. Five coils shape that different angle from $50^{\circ}$ to $90^{\circ}$ used in this study. As the result magnetic field was change quadratically by increasing the angle size. The best angle is between $60^{\circ}$ to $70^{\circ}$. The peak value is at $70^{\circ}$. If more than that the magnetic field will drop. The significant of this study is to get the most efficient angle for the electromagnet casing for the MR brakes.
\end{abstract}

\section{Introduction}

Nowadays there are two types of brake that are widely used. It is drum brake and disc brake. There are several problems these two types of brake. Drum brake has lower breaking efficiency and heat generation in drum is brake more [1]. The excessive heat can cause the brake fade [2]. Then disc brake being introduced to the industry, with several advantages compared to the drum brake. Although the disc brake is much better compared to the drum brake, but it also has its disadvantages. One of it, is, the efficiency of disc brake is too high which may cause skidding or wheel lock. Furthermore, during wet condition disc brake tended to decrease its efficiency. Considering the disadvantages of conventional brake system, thus there is a requirement to design a new braking system which is minimizing the effect mention.

Magneto rheological fluid is types of smart fluid that can change its rheological properties. A rapid change from liquid to semi solid state can occurs when a magnetic field induced to the fluids as shown in Fig. 1.

Magneto rheological fluid is a combination of magnetic particles suspended in a fluid medium. There are three component that made up MR fluid, iron particle, base fluid and additives. The sizes of the iron particle in the magneto rheological fluid are between 1-10 $\mu$-meters. The metal particle usually made up from carbonyl iron or powder iron or iron/

\footnotetext{
* Corresponding author: sitilydia@unikl.edu.my
} 
cobalt alloy, to achieve high magnetic saturation. When the magnetic field is applied the micro size particle will line up and a chain like structure will be form [3]. The viscosity of the fluid will be increase due to the alignment of the particles [4]. The base fluid commonly use is, hydrocarbon oil, mineral oil and silicon oil. The base fluid will act as the carrier fluid in which the metal is suspended. The base fluid should low in viscosity and not vary to the temperature. For the additive it should be high viscosity material such as grease or others thixotropic additives. The additives will act as suspending agent, thixotropers, friction modifier and anti-corrosion component [5].

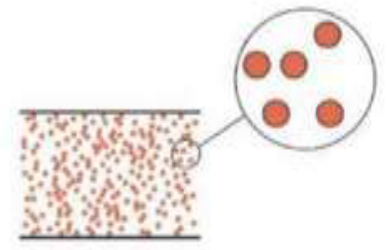

(a) Magneto rheological fluid without magnet field fluid with magnetic field

Fig. 1. Magneto Rheological Fluid behavior with or without magnet.

In recent year researcher has start developed MR brake [6], has comes out with Design and Consideration for an Automotive Magneto rheological Brake as shown in Fig. 2. A prototype of MR brakes is then built and tested and the experimental result show a good correlation with the finite element simulation predictions. However, the braking torque generated is still far less than conventional hydraulic brakes has design the brake using a Small Steel Roller and MR Fluid [7]. As the result shows the designed MR Brake have about $200 \%$ larger torque than the conventional one.

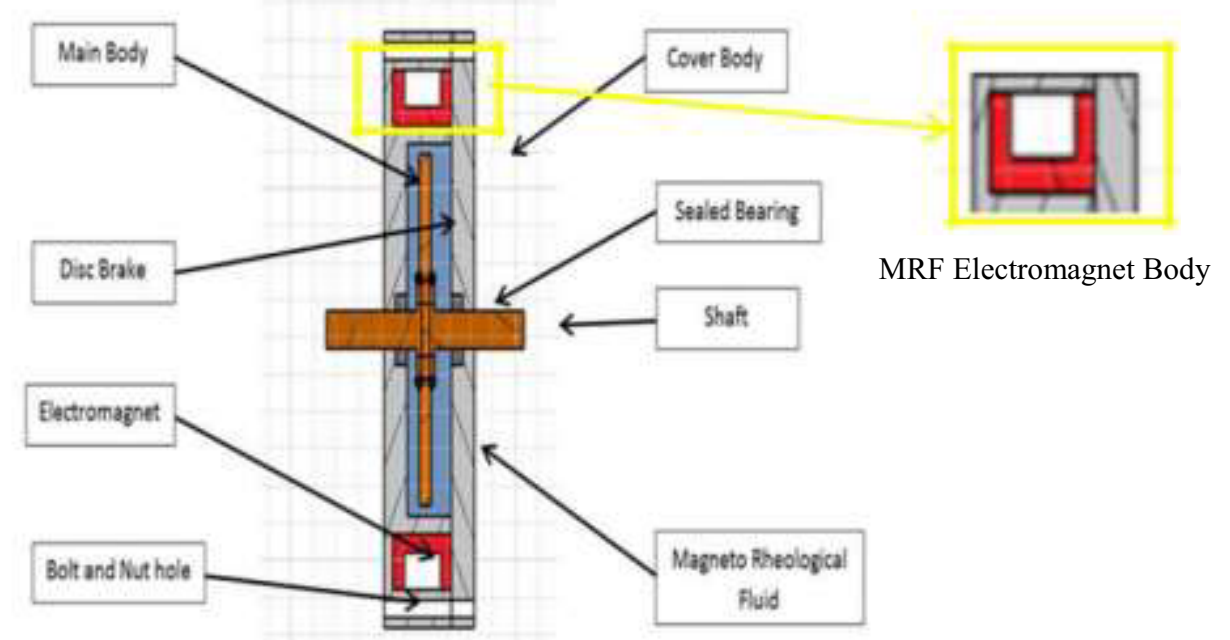

Fig. 2. Magneto Rheological Brake cross sectional area.

Figure 2 shows the cross sectional area for the MR brakes. The MR brake consists of a disc that being immersed into MR fluid in a closed contained and all the parts are made up of steel. The steel has been choosing because it has good electromagnetic conductivity. The highlighted part is the electromagnetic body which will be surrounding by copper wire to generate magnetic field to activate the MR fluid. The objective of this study is to analyze the effect of electromagnet body shapes on magnetic field induced. The material for each of the component is constant. The parameter that will be study is the angle of the electromagnet body and varies from $50^{\circ}$ to $90^{\circ}$. In order to design MRF brake system the 
magnetic circuit need to be analyzed. The variation of magnetic field inside the system is compulsory to study. Due to complicity in geometry the numerical method need to be used. In this paper will focusing on the shapes of the electromagnet. In which angle the electromagnet will give much greater magnetic field result.

\section{Methodology}

In this section will explain about the Magneto Rheological Brake electromagnet body design. Five different angles has been test, $50^{\circ}-90^{\circ}$, for the electromagnet body as shown in Fig. 3. FEMM software was used to analyze the magnetic field produced by the electromagnet.

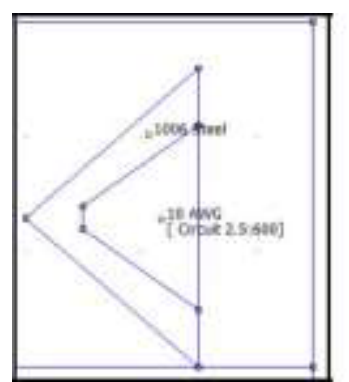

$50^{\circ}$ Design

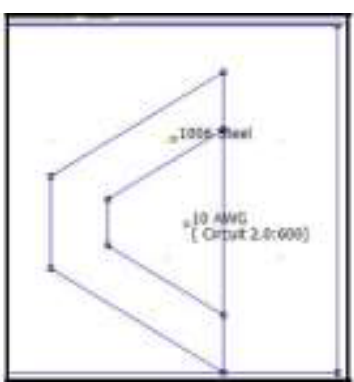

$60^{\circ}$ Design

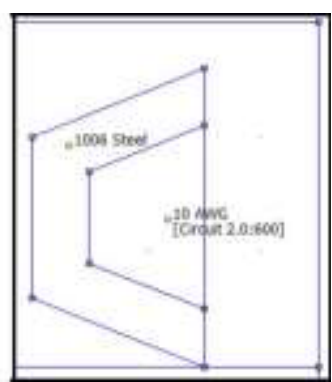

$70^{\circ}$ Design

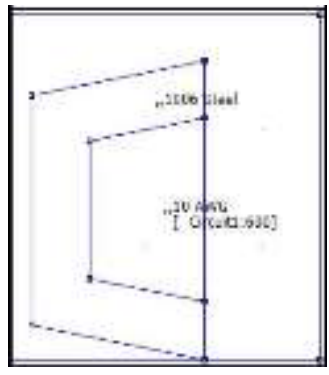

$80^{\circ}$ Design

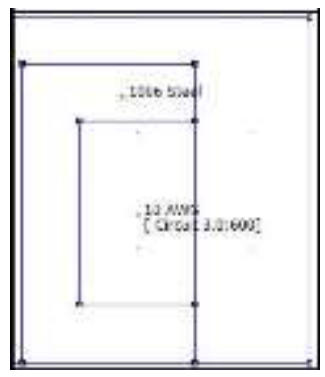

$90^{\circ}$ Design

Fig. 3. Electromagnet body design.

FEMM software used to discretize the geometry and solve the Maxwell equation numerically.The examined MRF Brake is characterized by the axial symmetry hence the field problem can be solved in the two-dimensional formulation in the Cartesian coordinate system. The computational domain for the magnetic field analysis is at the electromagnet body design with several different angles. Magnetostatic problems are problems in which the fields are time-invariant. In this case, the field intensity $(\mathrm{H})$ and flux density (B) must obey:

$$
\begin{aligned}
& \nabla \mathrm{X} H=J \\
& \nabla . B=0
\end{aligned}
$$

subject to a constitutive relationship between $\mathrm{B}$ and $\mathrm{H}$ for each material:

$$
B=\mu H
$$


If a material is nonlinear (e.g. saturating iron or alnico magnets), the permeability, $\mu$ is actually a function of B:

$$
\mu=\frac{B}{H(B)}
$$

Flux density is written in terms of the vector potential, A, as:

$$
B=\nabla \mathrm{X} A
$$

Now, this definition of B always satisfies (3.0). Then, (4.0) can be rewritten as:

$$
\nabla \mathrm{X}\left(\frac{1}{\mu(B)} \nabla \mathrm{X} A\right)=J
$$

For a linear isotropic material (and assuming the Coulomb gauge, $\nabla \cdot A=0$ ) reduces to :

$$
\frac{1}{\mu} \nabla^{2} A=J
$$

In any numerical method geometry model is the main input, same goes to the Finite Element. Figure 4(a) shows CAD model for MRF valve used in this research work. In order to reduce the computational time, the geometry was converted into 2 dimensional. Figure 4(b) shows the two dimensional valve after meshing process. Figure 5 shows the Magneto rheological brake assemble.

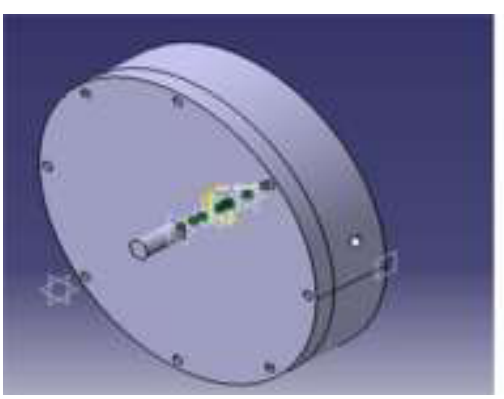

(a) CAD

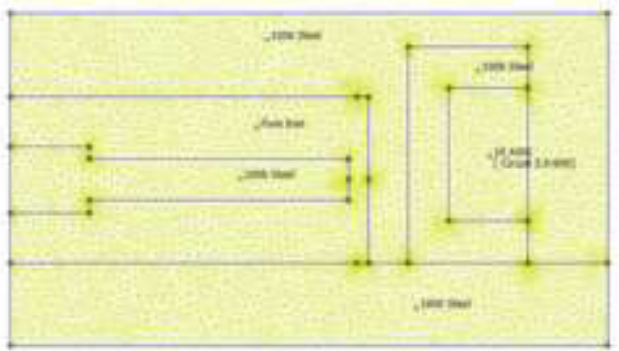

(b) Meshing

Fig. 4. CAD model for MR Brake and MRF Brake after mesh.

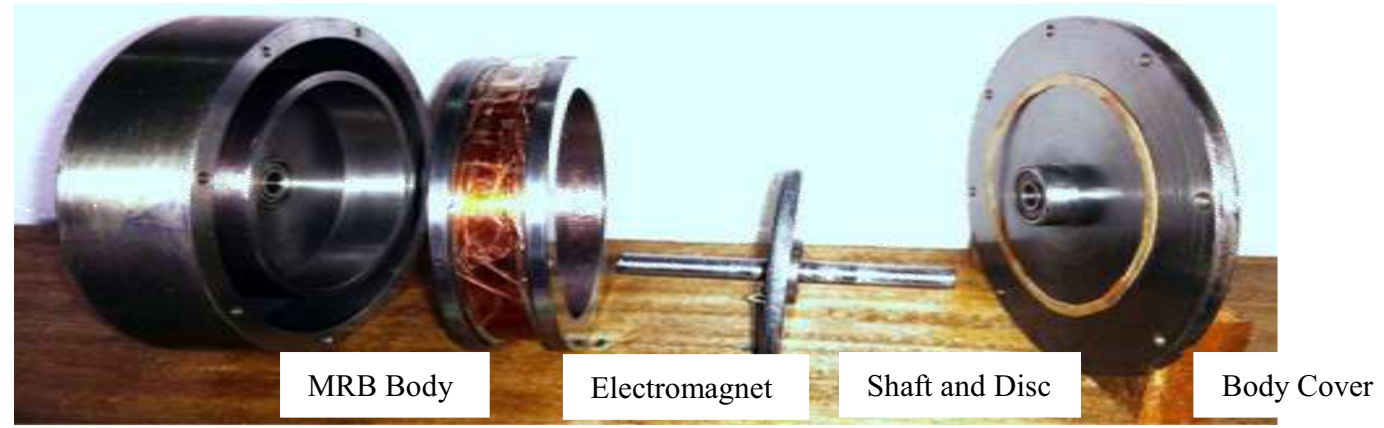

Fig. 5. Magneto rheological brake assemble. 


\section{Results and discussion}

The result from the FEMM can be shown in color contour for magnetic flux. The color will present the magnetic flux density and magnetic field strength. The plot value will be define along a define contour. From the result, it can define the B (magnitude of Flux Density). Figure 6 shows the result of the density plot for $50^{\circ}, 60^{\circ}, 70^{\circ}$ and $90^{\circ}$ designs. From the result, the purple region will show the highest Tesla value. Design $50^{\circ}, 60^{\circ}$ and $70^{\circ}$ has larger region with highest tesla value compared to the $90^{\circ}$. However when the tesla value at point 1 will show the highest value for tesla comes from $70^{\circ}$ degree design with value of 1.30498 Tesla.
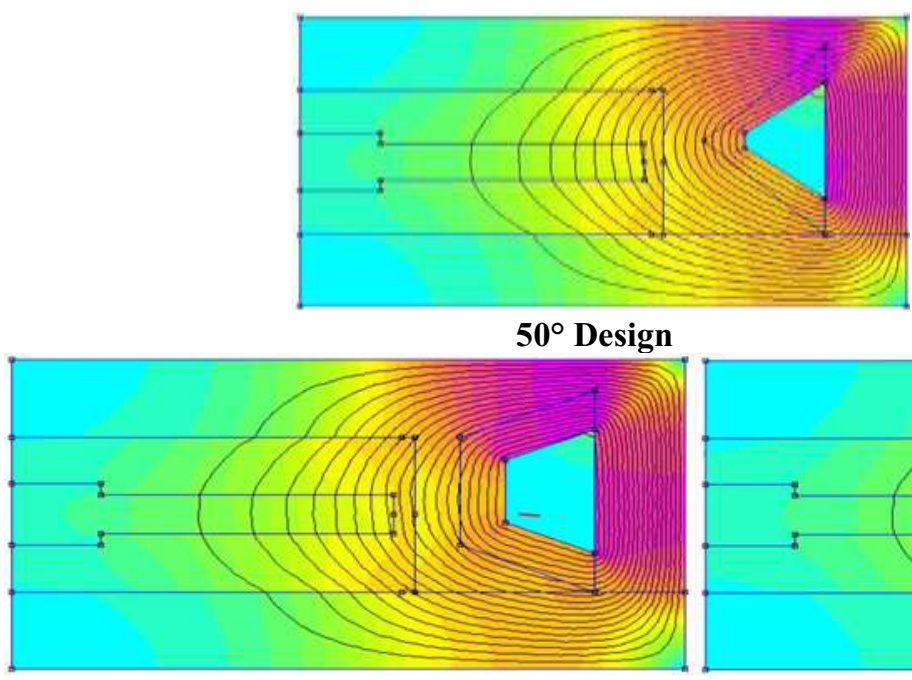

$70^{\circ}$ Design

Fig. 6. Magnetic Flux Density contour.

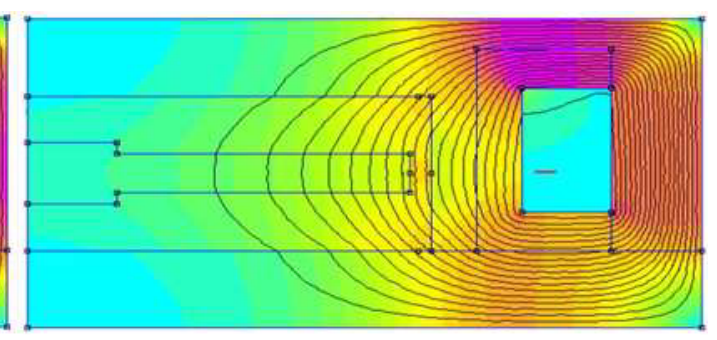

$90^{\circ}$ Design

Magnetic flux density is the amount of magnetic flux per unit area of a section, perpendicular to the direction of flux. From Fgiure 7, there are slightly increasing value of normal flux density (B.n) from 1.29024 tesla to 1.30303 tesla when the angle being increase from $50^{\circ}$ to $60^{\circ}$. From the density plot, the $60^{\circ}$ design has larger region that produce higher value of tesla. When the design changed to $70^{\circ}$ the value has reach to 1.30498 tesla, the highest value compared to the others design. The magnetic flux density plot shows that the $70^{\circ}$ angle will allow the magnetic flux to leading to the MR fluids more efficiently. The flux density slightly decreases when the angle changes to $80^{\circ}$ and $90^{\circ}$. As shown by the density plot, there are too small magnetic flux region being produces in the design.

The graph above shows the result of effect of current to the Tesla value. The test has been don at the point 1 . The current has been manipulated from 0.5 to 2.5 ampere. As the result from the three design chosen, $50^{\circ}, 70^{\circ}$ and $90^{\circ}$ it shows that the value of Tesla is increase due to the increasing current value. Although all the value is increasing, but the higher value comes from $70^{\circ}$ design. From the lower current supply, $0.5 \mathrm{~A}$, to the highest current, $2.5 \mathrm{~A}, 70^{\circ}$ design shows the higher value. This may cause by the geometry of the design that has good magnetic flux flow to the point 1 compared to the others. 


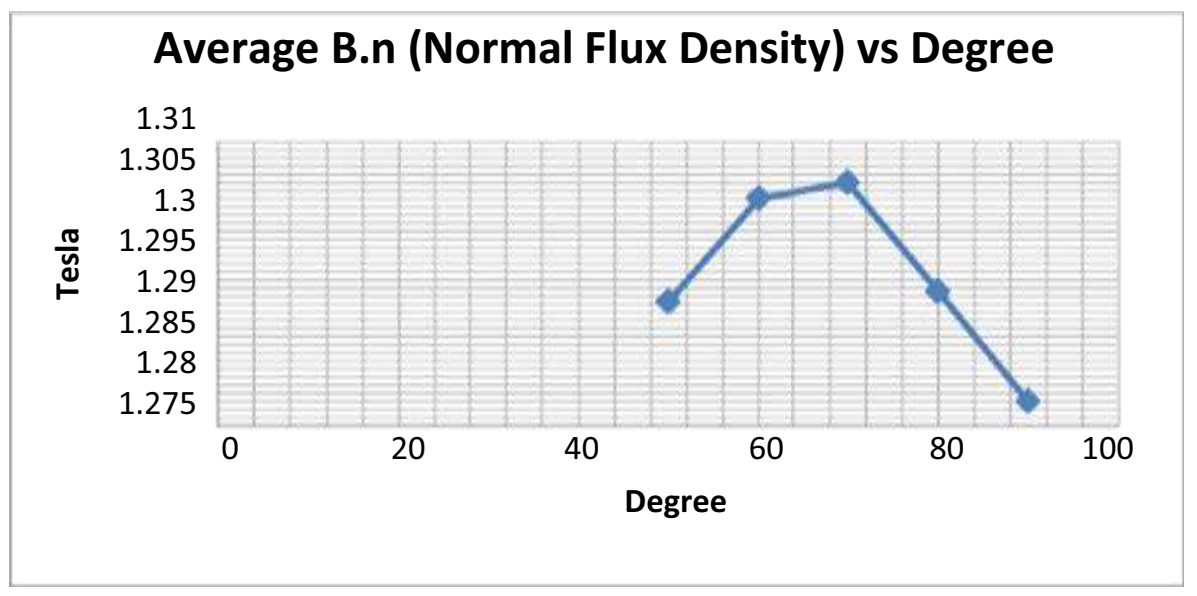

Fig. 7. B.n vs. Degree.

\section{Tesla vs Current}

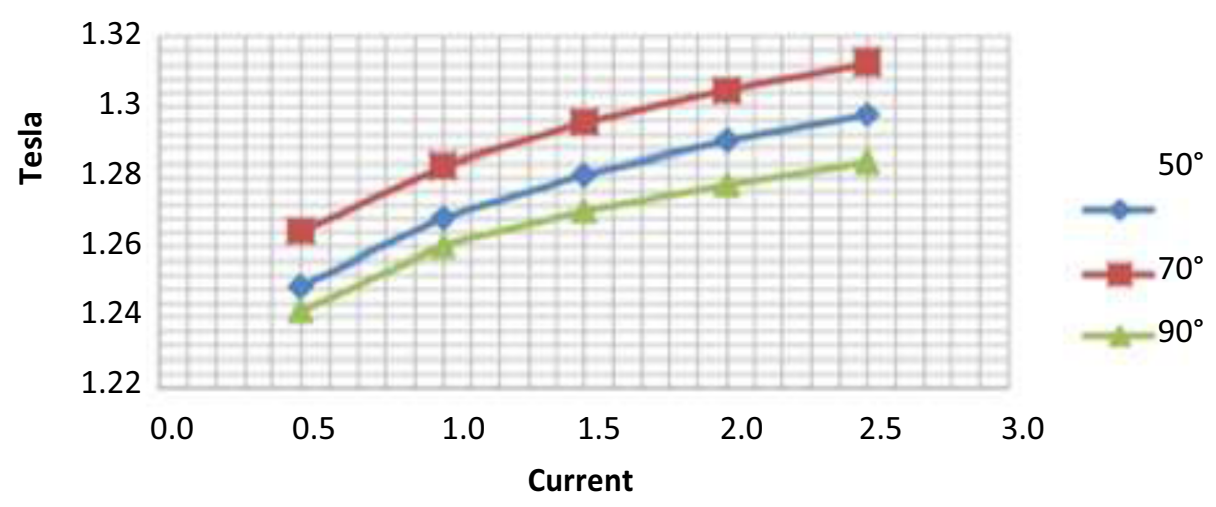

Fig. 8. Tesla vs. Current.

\section{Conclusions}

FEMM is the software is a solver to solve the governing equation for magnetic field distribution inside the domain. From the experiment above five different angle of design has been analysis using the FEMM. As the result, the good range for the electromagnet body design is between $50^{\circ}$ to $70^{\circ}$. If the design goes far than that the value of the magnetic field will be drop. As shown on graph 1 . Although the supply current been manipulated, the $70^{\circ}$ design still give the high value up to 1.31275 Tesla at 2.5 ampere.

\section{References}

1. Sriram T. Mutalik \& Tapan S. Kulkarni “Disc Oriented in Drum Brakes". International Journal of Emerging Technology and Advance Engineering, 2, Issues 10, (2012) 
2. Kannan M. Munisamy, Ramel Shafik "Disk Brake Design for Cooling Improvement Using Computational Fluid Dynamics (CFD)".IOP Conf. Series: earth And Environmental Science 16 (2013)

3. Luo Yiping, Xu Biao, Ren Hongjuan "Summarization of magneto-rheological fluid and its application in engineering". American Journal of Mechanics and Applications. 2, No. 1, 2014 pp. 1-5. doi: 10.11648/j.ajma.20140201.11

4. M. Kciuk, R. Turczyn " Propeties and Application of Magneto Rheological Fluid". Journal of Achievement in Material and Manufacturing Engineering, 18, (2006).

5. Deepak Baranwal, Dr. T.S. Deshmukh "MR-Fluid Technology and Its Application-A Review". International Journal of Emerging Technology and Advance Engineering, 2, (2012).

6. Kerem Karakoc, Edward J.Park, Afzal Suleman "Design consideration for an automotive magnetorheological brake".(2008).

7. Tran Hai NAM, Kyoung Kwan AHN "New approach to designing an MR brake using a small steel roller and MR fluid". Journal of Mechanical Science and Technology,23 $1911 \sim 1923$ (2009). 